\title{
Efeitos de Ambiente e de Heterose sobre o Ganho de Peso do Nascimento ao Desmame e sobre os Escores Visuais ao Desmame de Bovinos de Corte
}

\author{
Rossano André Dal-Farra1, Vanerlei Mozaquatro Roso², Flávio Schramm Schenkel ${ }^{3}$
}

\begin{abstract}
RESUMO - Os objetivos deste trabalho foram a obtenção de fatores de correção para efeitos de idade do bezerro, idade da vaca e data juliana do nascimento sobre o ganho de peso do nascimento à desmama (GND) e sobre os escores visuais de conformação (C), precocidade (P) e musculatura (M) e a estimação dos efeitos das heteroses materna e individual nessas características. Foram utilizados dados dos Programas de Melhoramento Genético Natura e Conexão Delta G, totalizando 187.610 bezerros puros e cruzas, nascidos no período de 1989 a 1999 em vários estados do Brasil. Os modelos estatísticos utilizados nas análises consideraram os efeitos fixos de grupo contemporâneo, idade do bezerro (regressão quadrática), idade da vaca (regressão polinomial quadrática-quadrática com nó aos 7 anos), data juliana de nascimento (regressão polinomial quadrática-quadrática-quadrática com nós aos 165 e 260 dias para GND, 150 e 240 dias para C, 165 e 270 dias para P e 155 e 260 dias para M) e heterozigoses materna e individual (regressões lineares). Todos os efeitos considerados no modelo influenciaram de forma significativa o GND e os escores de C, P e M. Os valores estimados de heterose materna e individual foram, respectivamente, $8,9 \%$ e $9,9 \%$ para GND, 10,0\% e 6,6\% para C, $8,5 \%$ e $4,8 \%$ para $\mathrm{P}$ e $10,9 \%$ e $5,0 \%$ para $\mathrm{M}$.
\end{abstract}

Palavras-chave: conformação, cruzamentos, fatores de ajustamento, musculatura, precocidade

\section{Environmental Effects and Heterosis on Weight Gain from Birth to Weaning and on Visual Scores at Weaning of Beef Cattle}

\begin{abstract}
The objectives of this study were to obtain adjustment factors for effects of calf age, dam age and calf Julian birth date on weight gain from birth to weaning (GND) and on visual scores of conformation (C), precocity (P), and muscling (M) and to estimate effects of maternal and individual heterosis on those traits. The analyzed records were from two breeding programs named Natura and Conexão Delta G, totalizing 187,610 purebred and crossbred Nellore x Angus and Nellore x Hereford calves. Calves were born between 1989 and 1999 in herds located in different states of Brazil. The statistical models applied to analyze GND and visual scores of C, P, and $\mathrm{M}$ included fixed effects of contemporary group, calf age (quadratic regression), dam age (quadratic-quadratic polynomial regression with junction point at seven years), calf Julian birth date (quadratic-quadratic-quadratic polynomial regression with junctions at 165 and 260 days for GND, 150 and 240 days for C, 165 and 270 days for P, and 155 and 260 days for M), and individual and maternal heterozygosity (linear regressions). All effects included in the model showed significant influence on GND and on visual scores of C, $\mathrm{P}$, and $\mathrm{M}$. The estimated values of maternal and individual heterosis were, respectively, $8.9 \%$ and $9.9 \%$ for GND; $10.0 \%$ and $6.6 \%$ for C; $8.5 \%$ and $4.8 \%$ for P; and $10.9 \%$ and $5.0 \%$ for $\mathrm{M}$.
\end{abstract}

Key Words: adjustment factors, conformation, crossbreeding, muscling, precocity

\section{Introdução}

Os programas de cruzamentos de bovinos de corte envolvendo raças zebuínas e taurinas têm sido utilizados como alternativa para obtenção de animais adequados às diferentes condições de produção no Brasil. A fim de otimizar estes benefícios, é necessário aliar às vantagens da heterose e da complementaridade uma adequada seleção dos animais, visando não apenas ao peso corporal (ou ganho de peso), mas também às características ligadas à morfologia e à estrutura corporal. Neste sentido, como complementação das medidas de peso corporal, vários programas de melhoramento genético têm avaliado, através de escores visuais, as características de conformação, precocidade e musculatura. Através dos escores de conformação, procura-se avaliar o potencial de produção de carcaças adequadas à produção de carne. Com base na observação da precocidade, avalia-se a capacidade de terminação com menor

\footnotetext{
${ }^{1}$ Professor-pesquisador da Universidade Luterana do Brasil - ULBRA. Av. Miguel Tostes, 101. Canoas/RS. CEP: 92420280. E.mail: rossanodf@uol.com.br

${ }^{2}$ GenSys Consultores Associados S/C Ltda, Porto Alegre/RS. E.mail: gensys@zaz.com.br

3 Pesquisador Associado - University of Guelph , Canada. E.mail: schenkel@uoguelph.ca
} 
peso e idade, enquanto, pelo escore de musculatura, avalia-se o desenvolvimento muscular do animal.

Alguns resultados de pesquisas sugerem que a seleção baseada nos escores de conformação, precocidade e musculatura pode ser efetiva, visto que as estimativas de herdabilidade obtidas para essas características são de moderada a média magnitude, semelhantes àquelas obtidas para ganho de peso (Pons et al., 1989; Roso \& Fries, 1995; Cardoso, 1999). Os estudos de Piccoli (1992), Pita et al. (1998) e Cardoso et al. (2001) em diferentes sistemas de criação confirmam a eficiência da seleção baseada nos escores visuais.

Para incrementar a acurácia do processo seletivo, os critérios de seleção devem ser adequadamente ajustados para os efeitos de ambiente. Existem vários trabalhos na literatura descrevendo os efeitos da idade do bezerro, idade da vaca e data juliana do nascimento sobre o ganho de peso. Entretanto, esta disponibilidade de resultados publicados não é verificada com relação aos escores visuais de conformação, precocidade e musculatura, o que justifica a realização de estudos para quantificar aqueles efeitos ambientais nesses escores.

Os objetivos do presente trabalho foram a obtenção de fatores de correção para o ganho de peso do nascimento à desmama e para os escores de conformação, precocidade e musculatura à desmama, e a estimação dos efeitos das heteroses materna e individual nessas características.

\section{Material e Métodos}

Os dados utilizados foram provenientes de 187.610 bezerros puros e cruzas Nelore x Angus e Nelore $\mathrm{x}$ Hereford de rebanhos comerciais participantes dos programas de melhoramento genético Natura e Conexão Delta G, nascidos no período de 1989 a 1999. Tais rebanhos estão distribuídos nos estados de Goiás, Mato Grosso, Mato Grosso do Sul, Minas Gerais, Paraná, Rio Grande do Sul, São Paulo e Tocantins. A composição racial dos animais de cada rebanho varia de acordo com o clima, condições nutricionais, manejo e objetivos comerciais de cada propriedade rural. Na região Sul do Brasil, predominam animais com maior fração das raças Angus e Hereford, enquanto que, nas demais regiões, os animais apresentam maior fração Nelore. A distribuição dos animais conforme a composição racial dos touros (pais) e das vacas (mães), expressas pela fração Nelore, é apresentada na Tabela 1.

As variáveis dependentes analisadas foram o ganho de peso do nascimento à desmama (GND, expresso em $\mathrm{kg}$ ) e os escores de conformação (C), precocidade $(\mathrm{P})$ e musculatura $(\mathrm{M})$ na desmama. Os escores foram avaliados utilizando-se uma escala de um a cinco de forma relativa dentro dos grupos de manejo, sendo que as notas mais altas indicam presença mais marcante da característica. Dos 187.610 bezerros avaliados para GND, aproximadamente $83 \%$ tinham avaliação para $\mathrm{C}, \mathrm{P}$ e $\mathrm{M}$.

Tabela 1 - Distribuição dos animais conforme classes de composição racial ${ }^{1}$ dos touros e das vacas expressas pela fração Nelore

Table 1 - Distribution of animals according to the breed composition classes ${ }^{1}$ of sires and dams

\begin{tabular}{|c|c|c|c|c|c|c|c|c|c|c|}
\hline \multirow[b]{2}{*}{$\begin{array}{l}\text { Touros } \\
\text { Sires }\end{array}$} & \multicolumn{10}{|c|}{$\begin{array}{l}\text { Vacas } \\
\text { Dams }\end{array}$} \\
\hline & 0,000 & 0,125 & 0,250 & 0,375 & 0,500 & 0,625 & 0,750 & 0,875 & 1,000 & Total \\
\hline 0,000 & 60533 & 10 & 278 & 417 & 6064 & 3469 & 3448 & 19 & 26381 & 100619 \\
\hline 0,125 & 0 & 0 & 3 & 25 & 0 & 0 & 1 & 0 & 5 & 34 \\
\hline 0,250 & 53 & 2 & 465 & 567 & 4925 & 24 & 168 & 2 & 948 & 7154 \\
\hline 0,375 & 671 & 40 & 2167 & 17931 & 5364 & 1220 & 377 & 18 & 13985 & 41773 \\
\hline 0,500 & 783 & 16 & 2213 & 115 & 1387 & 134 & 1491 & 0 & 3112 & 9251 \\
\hline 0,625 & 1512 & 0 & 58 & 19 & 824 & 1690 & 136 & 1 & 940 & 5180 \\
\hline 0,750 & 7056 & 26 & 213 & 43 & 240 & 84 & 364 & 4 & 15 & 8045 \\
\hline $0,875^{2}$ & 2279 & 3 & 400 & 58 & 3410 & 219 & 863 & 14 & 8308 & 15554 \\
\hline Total & 72887 & 97 & 5797 & 19175 & 22214 & 6840 & 6848 & 58 & 53694 & 187610 \\
\hline
\end{tabular}

\footnotetext{
1 Inclui touros ou vacas com composição racial maior ou igual ao valor da classe considerada e menor do que o valor da classe seguinte.

1 Includes sires or dams with breed composition greater than or equal to the value of the class considered and smaller than the value of the subsequent class.

${ }^{2}$ Não foram utilizados touros Nelore puros.

2 Purebred Nellore sires were not used.
}

R. Bras. Zootec., v.31, n.3, p.1350-1361, 2002 (suplemento) 
Para modelar os efeitos de ambiente, o GND e os escores de C, P e M foram analisados pelo Método dos Quadrados Mínimos, utilizando-se o procedimento GLM do SAS (1995). Inicialmente os modelos incluíram os efeitos de grupo contemporâneo (GC), idade do bezerro (IB), idade da vaca (IV) e data juliana do nascimento (DJ) como efeitos classificatórios, além da heterozigose materna (HM) e da heterozigose individual (HI) como covariáveis (efeito linear). As soluções obtidas através desses modelos foram utilizadas para determinar a ordem das regressões polinomiais, bem como os pontos de junção iniciais entre os segmentos das funções polinomiais segmentadas.

Os GCs foram formados por animais do mesmo sexo e ano de nascimento, pertencentes à mesma fazenda e grupo de manejo. As heterozigoses esperadas $(\mathrm{H})$ foram calculadas de acordo com a fórmula:

$$
\mathrm{H}=1-\sum_{\mathrm{i}=1}^{2} \mathrm{p}_{\mathrm{t}_{\mathrm{i}}} \mathrm{p}_{\mathrm{v}_{\mathrm{i}}}
$$

em que: $\mathrm{p}_{\mathrm{t}_{\mathrm{i}}}=$ fração da composição racial do t-ésimo touro para a raça $\mathrm{i}, \mathrm{i}=1,2$; e $\mathrm{p}_{\mathrm{v}_{\mathrm{i}}}=$ fração da composição racial da v-ésima vaca para a raça $i$, $i=1,2$.

As funções polinomiais utilizadas na modelagem das curvas dos efeitos de IB, IV e DJ foram:

IDADE DO BEZERRO (regressão quadrática)

$$
\mathrm{y}=\alpha+\mathrm{a}_{0}+\mathrm{a}_{1} \mathrm{x}+\mathrm{a}_{2} \mathrm{x}^{2}
$$

sendo: $\alpha=\mathrm{GC}+\mathrm{HM}+\mathrm{HI}+\mathrm{IV}+\mathrm{DJ}$, em que: $\mathrm{GC}=$ efeito fixo de grupo contemporâneo, $\mathrm{HM}, \mathrm{HI}=$ regressões lineares para as heterozigoses materna e individual, respectivamente, $\mathrm{IV}, \mathrm{DJ}=$ regressões polinomiais para a idade da vaca e para a data juliana de nascimento, respectivamente.

IDADE DA VACA (regressão polinomial quadrática-quadrática)

$$
\begin{array}{r}
y=\beta+a_{0}+a_{1} x+a_{2} x^{2}+b_{1} z \\
\text { sendo: } \beta=G C+H M+H I+I B+D J \\
z=0, \text { se } x \leq k ; \\
z=(x-k)^{2}, \text { se } x>k .
\end{array}
$$

$O$ efeito da idade da vaca foi modelado separadamente para machos e fêmeas, sendo IB a regressão polinomial para a idade do bezerro.

DATA JULIANA DE NASCIMENTO (regressão polinomial quadrática-quadrática-quadrática)

$$
\mathrm{y}=\lambda+\mathrm{a}_{0}+\mathrm{a}_{1} \mathrm{x}+\mathrm{a}_{2} \mathrm{x}^{2}+\mathrm{b}_{1} \mathrm{z}_{1}+\mathrm{b}_{2} \mathrm{z}_{2}
$$

em que: $\lambda=\mathrm{GC}+\mathrm{HM}+\mathrm{HI}+\mathrm{IB}+\mathrm{IV}$

$$
\begin{gathered}
\mathrm{z}_{1}=0, \text { se } \mathrm{x} \leq \mathrm{k}_{1} ; \\
\mathrm{z}_{1}=\left(\mathrm{x}-\mathrm{k}_{1}\right)^{2}, \text { se } \mathrm{x}>\mathrm{k}_{1} ;
\end{gathered}
$$

R. Bras. Zootec., v.31, n.3, p.1350-1361, 2002 (suplemento)

$$
\begin{gathered}
\mathrm{z}_{2}=0, \text { se } \mathrm{x} \leq \mathrm{k}_{2} ; \\
\mathrm{z}_{2}=\left(\mathrm{x}-\mathrm{k}_{2}\right)^{2}, \text { se } \mathrm{x}>\mathrm{k}_{2} .
\end{gathered}
$$

Nas funções polinomiais, y corresponde às variáveis dependentes GND, C, $\mathrm{P}$ e $\mathrm{M}$, enquanto $\mathrm{x}$ corresponde às variáveis independentes IB, IV e DJ. Os parâmetros das funções são representados por $\mathrm{a}_{0}$, $a_{1}, a_{2}, b_{1}$ e $b_{2}$. Os pontos de junção (nós) entre os segmentos das funções polinomiais são representados por $\mathrm{k}, \mathrm{k}_{1}$ e $\mathrm{k}_{2}$.

Os valores de $\mathrm{k}, \mathrm{k}_{1}$ e $\mathrm{k}_{2}$ foram, respectivamente, sete anos, 165 dias e 260 dias para GND, sete anos, 150 dias e 240 dias para C, sete anos, 165 dias e 270 dias para $\mathrm{P}$ e sete anos, 155 dias e 260 dias para $\mathrm{M}$. Os pontos de junção finais entre as funções polinomiais foram determinados de forma a maximizar o coeficiente de determinação do modelo.

As estimativas de heterose foram obtidas assumindo-se uma relação linear entre a heterose e a heterozigose esperada (Gregory\& Cundiff, 1980; Roso \& Fries, 2000).

Os fatores de correção (multiplicativos) para GND, C, P e M foram calculados utilizando-se como padrão sete anos para idade da vaca e 205 dias para idade do bezerro. Para a data juliana do nascimento foram utilizados os valores-padrão 45, 135, 225 e 315 dias para os bezerros nascidos entre 1 e 90,91 e 180 , 181 e 270 e 271 e 366 dias, respectivamente.

\section{Resultados e Discussão}

As médias, os desvios-padrão e as amplitudes das características analisadas são apresentados na Tabela 2.

Todos os efeitos considerados no modelo influenciaram significativamente o GND e os escores de C, $\mathrm{P}$ e $\mathrm{M}(\mathrm{P}<0,01)$, com exceção do efeito linear da data juliana do nascimento sobre os escores de $\mathrm{C}, \mathrm{P}$ e $\mathrm{M}$ $(\mathrm{P}>0,05)$.

As soluções obtidas para as covariáveis consideradas nas análises (IB, IV, DJ, HM e HI), bem como os coeficientes de determinação dos modelos para cada característica, são apresentados nas Tabelas 3, 4, 5 e 6 .

Os valores estimados para o GND e para os escores de C, $\mathrm{P}$ e $\mathrm{M}$ em função da idade do bezerro são apresentados na Figura 1. Na Figura 2 são apresentados os valores estimados para GND, C, P e $M$ em função da idade da vaca, enquanto que, na Figura 3, são apresentados os valores estimados para GND, C, P e M em função da data juliana do nascimento do bezerro. 
Tabela 2 - Médias, desvios-padrão e amplitudes dos ganhos de peso do nascimento à desmama (GND, em kg), escores de conformação (C), precocidade $(P)$ e musculatura $(M)$ à desmama, idades da vacas (IV, em anos), idades dos bezerros (IB, em dias), datas julianas dos nascimentos (DJ, em dias), heterozigoses maternas ( $\mathrm{HM}$, em \%) e heterozigoses individuais $(\mathrm{HI}$, em \%)

Table 2 - Means, standard deviations and ranges of weight gains from birth to weaning (GND, in $\mathrm{kg}$ ), and of visual scores of conformation (C), precocity $(P)$, and muscling (M), dam ages (IV, in years), calf ages (IB, in days), calf Julian birth dates (DJ, in days), maternal heterozigosities (HM, in \%), and individual heterozigosities (HI, in \%)

\begin{tabular}{lrcc}
\hline & $\begin{array}{c}\text { Média } \\
\text { Mean }\end{array}$ & $\begin{array}{c}\text { Desvio-padrão } \\
\text { Standard deviation }\end{array}$ & $\begin{array}{c}\text { Amplitude } \\
\text { Range }\end{array}$ \\
\hline GND & 148,49 & 38,44 & $33-357$ \\
C & 3,08 & 0,99 & $1-5$ \\
P & 3,28 & 0,94 & $1-5$ \\
M & 3,14 & 0,96 & $1-5$ \\
IV & 5,59 & 2,61 & $2-15$ \\
IB & 207,55 & 31,55 & $120-300$ \\
DJ & 263,67 & 54,30 & $1-366$ \\
HM & 0,17 & 0,25 & $0-1$ \\
HI & 0,41 & 0,36 & $0-1$ \\
\hline
\end{tabular}

Analisando-se as Figuras 1, 2 e 3, observa-se que, no comportamento geral, os efeitos da idade do bezerro, da idade da vaca e da data juliana do nascimento sobre o GND são muito similares àqueles encontrados sobre os escores de C, P e M. Resultados semelhantes foram obtidos por Cardoso (1999).

A idade à desmama apresentou efeito linear $\mathrm{e}$ quadrático sobre o GND e sobre os escores de C, P e M (Figura 1). Analisando ganho de peso médio diário, Paz (1997) e Teixeira (2000) encontraram efeitos semelhantes, alertando para o fato de que, em condições tropicais, apenas a regressão linear não parece suficiente para ajustar o GND para uma idade padrão à desmama. Este mesmo comportamento ocorre com relação aos escores de C, P e M, conforme demonstram os resultados do presente estudo.

A idade da vaca apresentou efeito semelhante sobre as quatro características analisadas (GND, C, $\mathrm{P}$ e $\mathrm{M}$ ), sendo que os dois segmentos quadráticos apresentaram efeito estatisticamente significativo $(\mathrm{P}<0,01)$. Conforme pode ser observado na Figura 2, o desempenho dos bezerros melhorou com o aumento da idade das vacas, atingindo o pico ao por volta dos sete anos. A partir dessa idade, o desempenho dos bezerros decresceu gradativamente. Com relação ao sexo dos bezerros, os machos foram superiores nas características GND, C e M, corroborando com os resultados obtidos por Fries (1996b), Paz (1997), Bocchi et al. (1999) e Teixeira (2000). Entretanto, para a característica $P$, as fêmeas apresentaram desempenho superior aos machos, com as maiores diferenças sendo observadas no período de maior produção leiteira das vacas.

A data juliana do nascimento apresentou três efeitos quadráticos ao longo do ano (regressão polinomial quadrática-quadrática-quadrática), causando expressiva variação no GND e nos escores de C, $\mathrm{P}$ e M entre períodos (Figura 3). Os animais nascidos no início da primavera foram os que apresentaram o melhor GND e obtiveram os melhores escores de C, $\mathrm{P}$ e M, provavelmente porque, nesta época do ano, as vacas atingem o pico da produção leiteira no momento de maior disponibilidade e qualidade forrageira. Analisando a característica ganho de peso médio diário, vários autores (Campos, 1989; Barcelos \& Lobato, 1992; Teixeira, 2000) obtiveram resultados semelhantes, evidenciando, assim como (Fries, 1996a), que a adoção de uma estação de monta que resulte em nascimentos no início da primavera proporciona os melhores resultados. No presente trabalho, a diferença em ganho de peso entre a melhor e a pior época foi de aproximadamente $25 \mathrm{~kg}$.

Os fatores de correção multiplicativos para o GND e para os escores de C, P e M, de acordo com a idade do bezerro, idade da vaca e data juliana de nascimento, são apresentados nas Tabelas 7, 8 e 9. Valores intermediários podem ser calculados a partir das soluções apresentadas nas Tabelas 3, 4, 5 e 6.

Ao analisar-se a magnitude dos fatores de correção, fica evidente a necessidade de ajustar-se para os efeitos de idade do bezerro, idade da vaca e data juliana do nascimento (ou incluí-los no modelo de avaliação), não apenas o GND, mas também os escores de $\mathrm{C}, \mathrm{P}$ e $\mathrm{M}$, objetivando correta avaliação genética dos animais. Para ilustração, considere dois bezerros machos (A e B), sendo o primeiro filho de uma vaca de dois anos e o segundo filho de uma vaca de 7 anos de idade. Na desmama, os dois bezerros têm escores de $\mathrm{C}$ iguais a 3,0. O bezerro A, por ser filho de uma vaca jovem, sofreria correção em C na ordem de 0,3996, equivalente a $13,3 \%$ de seu escore original, enquanto o escore do bezerro B não sofreria correção.

As heterozigoses materna e individual influenciaram significativamente o GND e os escores de C, P 
Tabela 3 - Soluções para ganho de peso do nascimento à desmama (GND)

Table 3 - Solutions for weight gain from birth to weaning (GND)

Fonte de variação

Soluções

Source of variation Solutions

\section{Constante}

$-64,845444 \pm 9,841916$

Intercept

Efeito linear da IV ${ }^{1}$ para machos

$14,572359 \pm 0,338780$

Linear effect of $I V^{l}$ for males

Efeito do $1^{\circ}$ segmento quadrático da IV para machos

$-1,076516 \pm 0,030286$

Effect of first quadratic segment of IV for males

Efeito do $2^{\mathrm{O}}$ segmento quadrático da IV para machos

$0,960207 \pm 0,049343$

Effect of second quadratic segment of IV for males

Efeito linear da IV para fêmeas

$12,026148 \pm 0,347675$

Linear effect of IV for females

Efeito do $1^{\circ}$ segmento quadrático da IV para fêmeas

$-0,878581 \pm 0,031205$

Effect of first quadratic segment of IV for females

Efeito do $2^{\circ}$ segmento quadrático da IV para fêmeas

$0,765490 \pm 0,051461$

Effect of second quadratic segment of IV for females

Efeito linear da $\mathrm{IB}^{2}$

$0,957840 \pm 0,020302$

Linear effect of $I B^{2}$

Efeito quadrático da IB

$-0,001181 \pm 0,000047$

Quadratic effect of $I B$

Efeito linear da $\mathrm{DJ}^{3}$

$-0,195373 \pm 0,015874$

Linear effect of $D J^{3}$

Efeito do $1^{\circ}$ segmento quadrático da DJ

$0,001610 \pm 0,000064$

Effect of first quadratic segment of $D J$

Efeito do $2^{\circ}$ segmento quadrático da DJ

$-0,004759 \pm 0,000115$

Effect of second quadratic segment of DJ

Efeito do $3^{\circ}$ segmento quadrático da DJ

$0,004279 \pm 0,000123$

Effect of third quadratic segment of $D J$

Efeito linear da $\mathrm{HM}^{4}$

Linear effect of $H M$

Efeito linear da $\mathrm{HI}^{5}$

$14,729548 \pm 0,364845$

Linear effect of $\mathrm{HI}$

$\mathrm{R}^{2}$ do modelo $=0,6917$

$R^{2}$ of model $=.6917$

$1 \mathrm{IV}=$ idade da vaca (IV = dam age)

$2 \mathrm{IB}=$ idade do bezerro $(I B=$ calf age $)$.

${ }^{3} \mathrm{DJ}=$ data juliana de nascimento ( $D J=$ calf Julian birth date).

${ }^{4} \mathrm{HM}=$ heterozigose materna (HM = maternal heterozigosity).

${ }^{5} \mathrm{H}=$ heterozigose individual $(\mathrm{HI}=$ individual heterozigosity $)$ 
Tabela 4 - Soluções para escore de conformação (C)

Table 4 - Solutions for conformation score (C)

Fonte de variação

Soluções

Source of variation

Solutions

\section{Constante}

Intercept

Efeito linear da IV ${ }^{1}$ para machos

$0,339604 \pm 0,260844$

Linear effect of $I V^{1}$ for males

Efeito do $1^{\mathrm{O}}$ segmento quadrático da IV para machos

$0,273133 \pm 0,013668$

Effect of first quadratic segment of IV for males

Efeito do $2^{\circ}$ segmento quadrático da IV para machos

$-0,019326 \pm 0,001225$

Effect of second quadratic segment of IV for males

Efeito linear da IV para fêmeas

$0,014763 \pm 0,002005$

Linear effect of IV for females

Efeito do $1^{\circ}$ segmento quadrático da IV para fêmeas

$0,262746 \pm 0,014066$

Effect of first quadratic segment of IV for females

Efeito do $2^{\mathrm{O}}$ segmento quadrático da IV para fêmeas

$-0,018304 \pm 0,001266$

Effect of second quadratic segment of IV for females

Efeito linear da IB ${ }^{2}$

Linear effect of $I B^{2}$

Efeito quadrático da IB

$0,013620 \pm 0,002106$

Quadratic effect of IB

Efeito linear da $\mathrm{DJ}^{3}$

$0,023643 \pm 0,000836$

Linear effect of $D J^{3}$

Efeito do $1^{\circ}$ segmento quadrático da DJ

$-0,000037 \pm 0,000001$

Effect of first quadratic segment of DJ

Efeito do $2^{\circ}$ segmento quadrático da DJ

$-0,002453 \pm 0,001645$

Effect of second quadratic segment of DJ

Efeito do $3^{\circ}$ segmento quadrático da DJ

$0,000024 \pm 0,000007$

Effect of third quadratic segment of DJ

Efeito linear da $\mathrm{HM}^{4}$

$-0,000074 \pm 0,000009$

Linear effect of $H M$

Efeito linear da $\mathrm{HI}^{5}$

$0,000064 \pm 0,000005$

Linear effect of $H I$

$\mathrm{R}^{2}$ do modelo $=0,3682$

$R^{2}$ of model $=.3682$

\footnotetext{
${ }^{1} \mathrm{IV}=$ idade da vaca $(\mathrm{IV}=$ dam age $)$.

$2 \mathbb{B}=$ idade do bezerro $(I B=$ calf age $)$.

${ }^{3} \mathrm{DJ}=$ data juliana de nascimento $(\mathrm{DJ}=$ calf Julian birth date).

${ }^{4} \mathrm{HM}=$ heterozigose materna ( $H M=$ maternal heterozigosity).

${ }^{5} \mathrm{H}=$ heterozigose individual $(\mathrm{HI}=$ individual heterozigosity).
}

$0,309609 \pm 0,016373$

$0,203681 \pm 0,014238$ 
Tabela 5 - Soluções para escore de precocidade $(P)$

Table 5 - Solutions for precocity score $(P)$

\begin{tabular}{|c|c|}
\hline $\begin{array}{l}\text { Fonte de variação } \\
\text { Source of variation }\end{array}$ & $\begin{array}{l}\text { Soluções } \\
\text { Solutions }\end{array}$ \\
\hline Constante & $-0,371682 \pm 0,261107$ \\
\hline \multicolumn{2}{|l|}{ Intercept } \\
\hline Efeito linear da IV ${ }^{1}$ para machos & $0,254197 \pm 0,014092$ \\
\hline \multicolumn{2}{|l|}{ Linear effect of IV for males } \\
\hline Efeito do $1^{\circ}$ segmento quadrático da IV para machos & $-0,018040 \pm 0,001263$ \\
\hline \multicolumn{2}{|l|}{ Effect of first quadratic segment of IV for males } \\
\hline Efeito do $2^{\circ}$ segmento quadrático da IV para machos & $0,014638 \pm 0,002066$ \\
\hline \multicolumn{2}{|l|}{ Effect of second quadratic segment of IV for males } \\
\hline Efeito linear da IV para fêmeas & $0,271393 \pm 0,014511$ \\
\hline \multicolumn{2}{|l|}{ Linear effect of IV for females } \\
\hline Efeito do $1^{\circ}$ segmento quadrático da IV para fêmeas & $-0,018786 \pm 0,001306$ \\
\hline \multicolumn{2}{|l|}{ Effect of first quadratic segment of IV for females } \\
\hline Efeito do $2^{\circ}$ segmento quadrático da IV para fêmeas & $0,014135 \pm 0,002171$ \\
\hline \multicolumn{2}{|l|}{ Effect of second quadratic segment of IV for females } \\
\hline Efeito linear da $\mathrm{IB}^{2}$ & $0,023260 \pm 0,000856$ \\
\hline \multicolumn{2}{|l|}{ Linear effect of $I B^{2}$} \\
\hline Efeito quadrático da IB & $-0,000039 \pm 0,000002$ \\
\hline \multicolumn{2}{|l|}{ Quadratic effect of IB } \\
\hline Efeito linear da $\mathrm{DJ}^{3}$ & $-0,001615 \pm 0,001154$ \\
\hline \multicolumn{2}{|l|}{ Linear effect of $D J^{3}$} \\
\hline Efeito do $1^{\circ}$ segmento quadrático da DJ & $0,000015 \pm 0,000004$ \\
\hline \multicolumn{2}{|l|}{ Effect of first quadratic segment of DJ } \\
\hline Efeito do $2^{\circ}$ segmento quadrático da DJ & $-0,000052 \pm 0,000006$ \\
\hline \multicolumn{2}{|l|}{ Effect of second quadratic segment of DJ } \\
\hline Efeito do $3^{\circ}$ segmento quadrático da DJ & $0,000070 \pm 0,000005$ \\
\hline \multicolumn{2}{|l|}{ Effect of third quadratic segment of DJ } \\
\hline Efeito linear da $\mathrm{HM}^{4}$ & $0,277618 \pm 0,016880$ \\
\hline \multicolumn{2}{|l|}{ Linear effect of $H M$} \\
\hline Efeito linear da $\mathrm{HI}^{5}$ & $0,157401 \pm 0,014661$ \\
\hline \multicolumn{2}{|l|}{ Linear effect of $H I$} \\
\hline \multicolumn{2}{|l|}{$\mathrm{R}^{2}$ do modelo $=0,2520$} \\
\hline$R^{2}$ of model $=.2520$ & \\
\hline
\end{tabular}

${ }_{1} \mathrm{IV}=$ idade da vaca $(\mathrm{IV}=$ dam age).

${ }^{2} \mathrm{~B}=$ idade do bezerro $(I B=$ calf age $)$.

${ }^{3} \mathrm{DJ}=$ data juliana de nascimento $(\mathrm{DJ}=$ calf Julian birth date $)$.

${ }^{4} \mathrm{HM}=$ heterozigose materna $(\mathrm{HM}=$ maternal heterozigosity $)$.

${ }^{5} \mathrm{H}=$ heterozigose individual $(\mathrm{HI}=$ individual heterozigosity). 
Tabela 6 - Soluções para escore de musculatura (M)

Table 6 - Solutions for muscling score (M)

Fonte de variação

Soluções

Source of variation

Solutions

\section{Constante}

$0,036917 \pm 0,259713$

Intercept

Efeito linear da IV ${ }^{1}$ para machos

$0,296912 \pm 0,014172$

Linear effect of $I V^{1}$ for males

Efeito do $1^{\circ}$ segmento quadrático da IV para machos

$-0,021011 \pm 0,001270$

Effect of first quadratic segment of IV for males

Efeito do $2^{\mathrm{O}}$ segmento quadrático da IV para machos

$0,016799 \pm 0,002078$

Effect of second quadratic segment of IV for males

Efeito linear da IV para fêmeas

$0,288376 \pm 0,014593$

Linear effect of IV for females

Efeito do $1^{\circ}$ segmento quadrático da IV para fêmeas

$-0,020439 \pm 0,001314$

Effect of first quadratic segment of IV for females

Efeito do $2^{\circ}$ segmento quadrático da IV para fêmeas

$0,016399 \pm 0,002183$

Effect of second quadratic segment of IV for females

Efeito linear da $\mathrm{IB}^{2}$

$0,020101 \pm 0,000858$

Linear effect of $I B^{2}$

Efeito quadrático da IB

$-0,000032 \pm 0,000002$

Quadratic effect of $I B$

Efeito linear da $\mathrm{DJ}^{3}$

$-0,000556 \pm 0,001129$

Linear effect of $D J^{3}$

Efeito do $1^{\mathrm{O}}$ segmento quadrático da DJ

$0,000013 \pm 0,000004$

Effect of first quadratic segment of DJ

Efeito do $2^{\circ}$ segmento quadrático da DJ

$-0,000053 \pm 0,000006$

Effect of second quadratic segment of $D J$

Efeito do $3^{\circ}$ segmento quadrático da DJ

$0,000063 \pm 0,000005$

Effect of third quadratic segment of $D J$

Efeito linear da $\mathrm{HM}^{4}$

$0,342694 \pm 0,016969$

Linear effect of $H M$

Efeito linear da $\mathrm{HI}^{5}$

$0,156395 \pm 0,014723$

Linear effect of $H I$

$\mathrm{R}^{2}$ do modelo: 0,2597

$R^{2}$ of model $=.2597$

\footnotetext{
${ }^{1} \mathrm{IV}=$ idade da vaca $(I V=$ dam age).

$2 \mathrm{BB}=$ idade do bezerro (IB = calf age).

${ }^{3} \mathrm{DJ}=$ data juliana de nascimento $(\mathrm{DJ}=$ calf Julian birth date).

${ }^{4} \mathrm{HM}=$ heterozigose materna (HM = maternal heterozigosity).

${ }^{5} \mathrm{H}=$ heterozigose individual $(\mathrm{HI}=$ individual heterozigosity).
} 


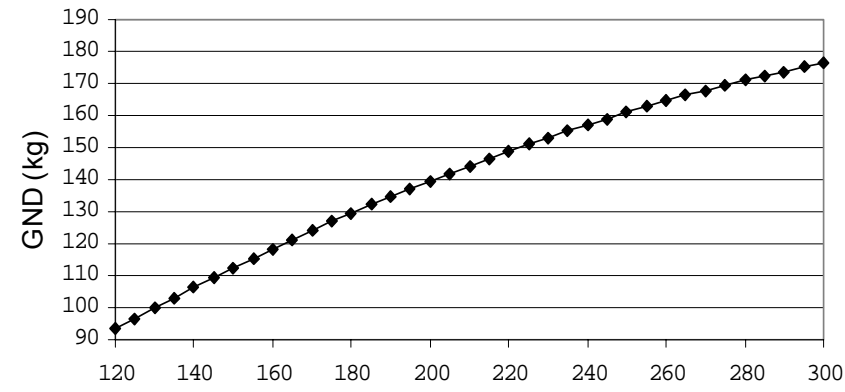

Idade do bezerro (dias)

Calf age (days)

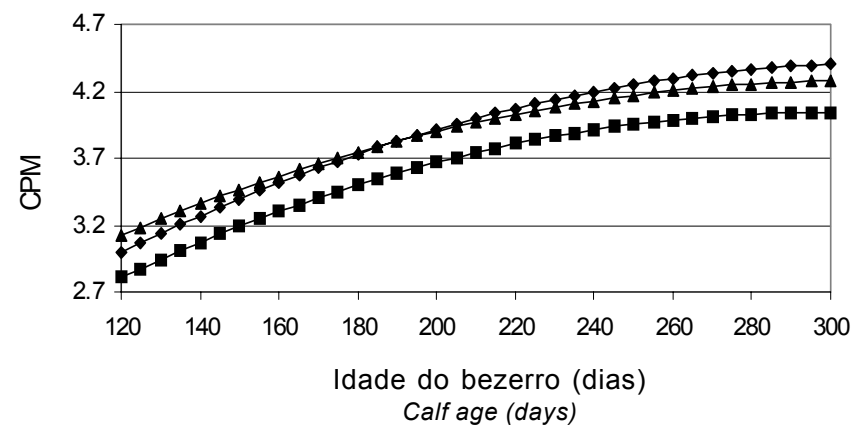

$\rightarrow C \rightarrow P \rightarrow M$

Figura 1 - Valores estimados para o ganho de peso do nascimento à desmama (GND) e para os escores de conformação $(C)$, precocidade $(P)$ e musculatura $(M)$ em função da idade do bezerro.

Figure 1 - Estimated values for weight gain from birth to weaning (GND) and for conformation (C), precocity (P), and muscling (M) scores as a function of calf age.
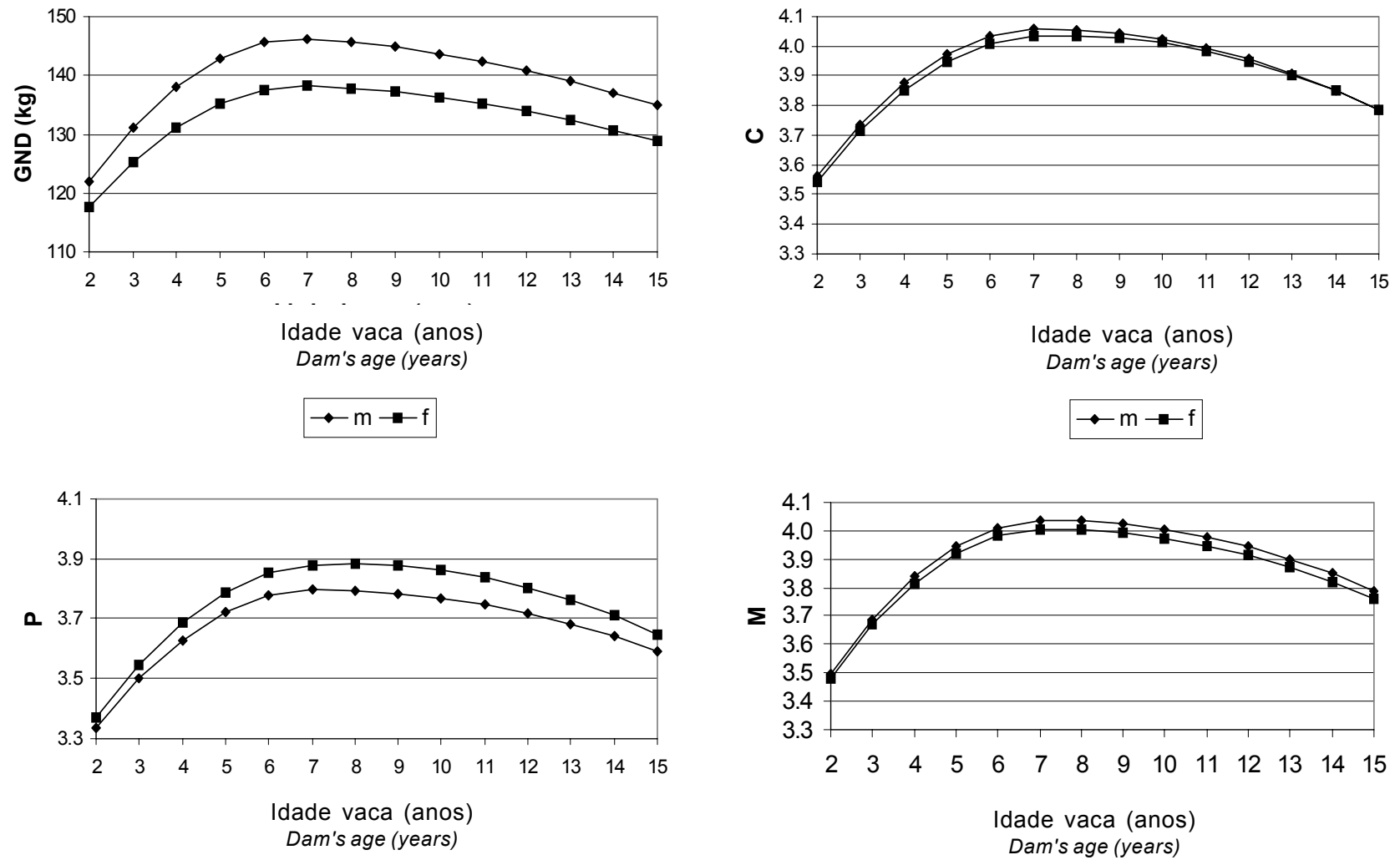

Figura 2 - Valores estimados para o ganho de peso do nascimento à desmama (GND) e para os escores de conformação $(C)$, precocidade $(P)$ e musculatura $(M)$, para machos $(m)$ e fêmeas (f), em função da idade da vaca.

Figure 2 - Estimated values for weight gain from birth to weaning (GND) and for conformation (C), precocity (P) and muscling (M) scores, for male $(m)$ and females ( $f$ ), as a function of dam age. 
Tabela 7 - Fatores de correção multiplicativos para o ganho de peso do nascimento à desmama (GND) e para os escores visuais de conformação $(C)$, precocidade $(P)$ e musculatura (M), conforme a idade do bezerro (IB)

Table 7 - Adjustment factors for weight gain from birth to weaning (GND) and for visual scores of conformation $(C)$, precocity $(P)$, and muscling $(M)$ for different calf ages

\begin{tabular}{lcccc}
\hline IB (dias) & GND & $\mathrm{C}$ & $\mathrm{P}$ & $\mathrm{M}$ \\
$I B$ (days) & & & & \\
\hline 120 & 1,5018 & 1,3088 & 1,3013 & 1,2464 \\
130 & 1,4059 & 1,2520 & 1,2450 & 1,2026 \\
140 & 1,3244 & 1,2026 & 1,1962 & 1,1639 \\
150 & 1,2544 & 1,1595 & 1,1537 & 1,1296 \\
160 & 1,1937 & 1,1216 & 1,1167 & 1,0993 \\
170 & 1,1407 & 1,0883 & 1,0843 & 1,0723 \\
180 & 1,0942 & 1,0589 & 1,0560 & 1,0484 \\
190 & 1,0531 & 1,0331 & 1,0312 & 1,0272 \\
200 & 1,0167 & 1,0103 & 1,0097 & 1,0085 \\
210 & 0,9842 & 0,9903 & 0,9910 & 0,9920 \\
220 & 0,9553 & 0,9729 & 0,9749 & 0,9776 \\
230 & 0,9294 & 0,9577 & 0,9611 & 0,9651 \\
240 & 0,9062 & 0,9446 & 0,9496 & 0,9543 \\
250 & 0,8854 & 0,9335 & 0,9401 & 0,9452 \\
260 & 0,8667 & 0,9242 & 0,9325 & 0,9377 \\
270 & 0,8500 & 0,9166 & 0,9268 & 0,9316 \\
280 & 0,8350 & 0,9106 & 0,9229 & 0,9270 \\
290 & 0,8217 & 0,9063 & 0,9206 & 0,9239 \\
300 & 0,8098 & 0,9034 & 0,9202 & 0,9220 \\
\hline
\end{tabular}

e $\mathrm{M}(\mathrm{P}<0,01)$. Os valores estimados de heterose, associados às heterozigoses materna e individual, foram, respectivamente: $8,9 \%(13,2 \mathrm{~kg})$ e $9,9 \%(14,7$ kg) para GND; $10,0 \%$ e $6,6 \%$ para $C ; 8,5 \%$ e $4,8 \%$ para $\mathrm{P}$ e $10,9 \%$ e $5,0 \%$ para $\mathrm{M}$.

Valores elevados de heterose para características relacionadas ao peso e à desmama foram relatados por vários autores. Roso e Fries (2000), analisando o ganho de peso pré-desmama de animais Angus x Nelore, obtiveram 9,1\% (13,3 kg) para heterose individual e $15,5 \%(22,6 \mathrm{~kg})$ para heterose materna, enquanto Fries et al. (2000), analisando resultados de cruzamentos entre zebuínos e taurinos, obtiveram valores de 9,8\% para heterose individual e 13,1\% para heterose materna sobre o peso à desmama. Teixeira (2000), utilizando três modelos diferentes, obteve valores entre $6,2 \%$ e $21,8 \%$ para heterose individual e 8,1 e 11,2\% para heterose materna sobre o ganho médio diário pré-desmama.

As heteroses materna e individual foram similares entre os escores de C, P e M. Não foram encontradas referências na literatura que possibilitassem comparação direta com os resultados obtidos neste trabalho. Entretanto, resultados de heterose

Tabela 8 - Fatores de correção multiplicativos para o ganho de peso do nascimento à desmama (GND) e para os escores visuais de conformação $(\mathrm{C})$, precocidade $(\mathrm{P})$ e musculatura $(\mathrm{M})$, conforme a idade da vaca (IV), para machos e para fêmeas

Table 8 - Adjustment factors for weight gain from birth to weaning (GND), and for visual scores of conformation (C), precocity (P), and muscling (M) for different dam ages (IV), for males and females

\begin{tabular}{|c|c|c|c|c|c|c|c|c|}
\hline \multirow[b]{2}{*}{$\begin{array}{l}\text { IV (anos) } \\
\text { IV (years) }\end{array}$} & \multicolumn{2}{|c|}{ GND } & \multicolumn{2}{|c|}{$\mathrm{C}$} & \multicolumn{2}{|c|}{$\mathrm{P}$} & \multicolumn{2}{|c|}{$\mathrm{M}$} \\
\hline & $\begin{array}{c}\text { Machos } \\
\text { Males }\end{array}$ & $\begin{array}{l}\text { Fêmeas } \\
\text { Females }\end{array}$ & $\begin{array}{c}\text { Machos } \\
\text { Males }\end{array}$ & $\begin{array}{l}\text { Fêmeas } \\
\text { Females }\end{array}$ & $\begin{array}{c}\text { Machos } \\
\text { Males }\end{array}$ & $\begin{array}{l}\text { Fêmeas } \\
\text { Females }\end{array}$ & $\begin{array}{c}\text { Machos } \\
\text { Males }\end{array}$ & $\begin{array}{l}\text { Fêmeas } \\
\text { Females }\end{array}$ \\
\hline 2 & 1,1880 & 1,1640 & 1,1332 & 1,1322 & 1,1320 & 1,1457 & 1,1471 & 1,1430 \\
\hline 3 & 1,1095 & 1,0973 & 1,0819 & 1,0822 & 1,0810 & 1,0906 & 1,0900 & 1,0875 \\
\hline 4 & 1,0561 & 1,0510 & 1,0450 & 1,0459 & 1,0444 & 1,0507 & 1,0493 & 1,0479 \\
\hline 5 & 1,0219 & 1,0207 & 1,0199 & 1,0210 & 1,0195 & 1,0234 & 1,0218 & 1,0211 \\
\hline 6 & 1,0038 & 1,0042 & 1,0052 & 1,0059 & 1,0050 & 1,0068 & 1,0057 & 1,0055 \\
\hline 7 & 1,0000 & 1,0000 & 1,0000 & 1,0000 & 1,0000 & 1,0000 & 1,0000 & 1,0000 \\
\hline 8 & 1,0040 & 1,0027 & 1,0005 & 0,9996 & 1,0004 & 0,9991 & 1,0003 & 1,0004 \\
\hline 9 & 1,0096 & 1,0069 & 1,0031 & 1,0014 & 1,0026 & 1,0005 & 1,0027 & 1,0028 \\
\hline 10 & 1,0168 & 1,0127 & 1,0080 & 1,0054 & 1,0066 & 1,0042 & 1,0071 & 1,0072 \\
\hline 11 & 1,0256 & 1,0203 & 1,0151 & 1,0118 & 1,0123 & 1,0103 & 1,0136 & 1,0135 \\
\hline 12 & 1,0363 & 1,0296 & 1,0246 & 1,0206 & 1,0199 & 1,0189 & 1,0223 & 1,0220 \\
\hline 13 & 1,0488 & 1,0407 & 1,0366 & 1,0319 & 1,0294 & 1,0300 & 1,0332 & 1,0327 \\
\hline 14 & 1,0634 & 1,0538 & 1,0512 & 1,0459 & 1,0410 & 1,0439 & 1,0466 & 1,0457 \\
\hline 15 & 1,0801 & 1,0690 & 1,0688 & 1,0628 & 1,0548 & 1,0609 & 1,0625 & 1,0612 \\
\hline
\end{tabular}


individual obtidos com características de carcaça teoricamente relacionadas com $\mathrm{C}, \mathrm{P}$ e M, avaliadas após o abate, relatados por Restle et al. (2000), mostram magnitudes de heterose de baixa à moderada, semelhantes às estimadas para $\mathrm{C}, \mathrm{P}$ e $\mathrm{M}$ no presente estudo. Os autores referidos, trabalhando com animais mestiços Charolês x Nelore abatidos aos 24 meses de idade, encontraram valores de heterose individual de $17,4 \%$ para espessura de gordura, 8,3\% para conformação de carcaça, $6,0 \%$ para espessura de coxão, $4,6 \%$ para perímetro de braço, $4,1 \%$ para comprimento de perna, $3,7 \%$ para comprimento de carcaça, $3,4 \%$ para porcentagem de gordura na carcaça, $3,1 \%$ para rendimento de carcaça e $0,6 \%$ para porcentagem de músculo.

A heterose materna para os escores C, P e M foi substancialmente superior à heterose individual e de magnitude semelhante à heterose materna para GND. Entretanto, a heterose individual dos escores foi bastante inferior àquela estimada para GND.

A magnitude dos valores de heterose para os escores de C, P e M foi expressiva (por volta de $10 \%$ e $5 \%$ para as heteroses materna e individual, respectivamente), indicando que ambas heteroses deveriam ser consideradas nas avaliações genéticas dos animais para C, P e M, juntamente com os efeitos ambientais de idade do bezerro, idade da vaca e data juliana do nascimento do bezerro.
Tabela 9 - Fatores de correção multiplicativa do ganho de peso do nascimento à desmama (GND) e dos escores visuais de conformação $(C)$, precocidade $(P)$ e musculatura (M), conforme a data juliana do nascimento (DJ)

Table 9 - Adjustment factors for weight gain from birth to weaning (GND) and for visual scores of conformation $(C)$, precocity $(P)$, and muscling $(M)$ for different calf's Julian birth dates (DJ)

\begin{tabular}{lcccc}
\hline DJ (dias) & GND & $\mathrm{C}$ & $\mathrm{P}$ & $\mathrm{M}$ \\
$D J$ (days) & & & & \\
\hline 1 & 0,9597 & 0,9854 & 0,9894 & 0,9968 \\
15 & 0,9772 & 0,9926 & 0,9944 & 1,0021 \\
30 & 0,9913 & 0,9977 & 0,9981 & 1,0018 \\
45 & 1,0000 & 1,0000 & 1,0000 & 1,0000 \\
60 & 1,0031 & 0,9995 & 1,0000 & 0,9966 \\
75 & 1,0005 & 0,9961 & 0,9982 & 0,9916 \\
90 & 0,9923 & 0,9899 & 0,9945 & 0,9852 \\
105 & 1,0441 & 1,0262 & 1,0165 & 1,0206 \\
120 & 1,0244 & 1,0143 & 1,0091 & 1,0110 \\
135 & 1,0000 & 1,0000 & 1,0000 & 1,0000 \\
150 & 0,9717 & 0,9834 & 0,9893 & 0,9878 \\
165 & 0,9402 & 0,9687 & 0,9771 & 0,9756 \\
180 & 0,9128 & 0,9593 & 0,9664 & 0,9675 \\
195 & 1,0104 & 0,9933 & 1,0007 & 0,9956 \\
210 & 1,0005 & 0,9940 & 0,9982 & 0,9957 \\
225 & 1,0000 & 1,0000 & 1,0000 & 1,0000 \\
240 & 1,0088 & 1,0114 & 1,0060 & 1,0086 \\
255 & 1,0275 & 1,0249 & 1,0164 & 1,0217 \\
270 & 1,0539 & 1,0370 & 1,0315 & 1,0381 \\
285 & 0,9636 & 0,9844 & 0,9811 & 0,9799 \\
300 & 0,9833 & 0,9930 & 0,9924 & 0,9912 \\
315 & 1,0000 & 1,0000 & 1,0000 & 1,0000 \\
330 & 1,0135 & 1,0054 & 1,0037 & 1,0063 \\
345 & 1,0234 & 1,0092 & 1,0034 & 1,0098 \\
360 & 1,0295 & 1,0112 & 0,9991 & 1,0107 \\
365 & 1,0309 & 1,0115 & 1,0009 & 1,0104 \\
\hline & & & &
\end{tabular}

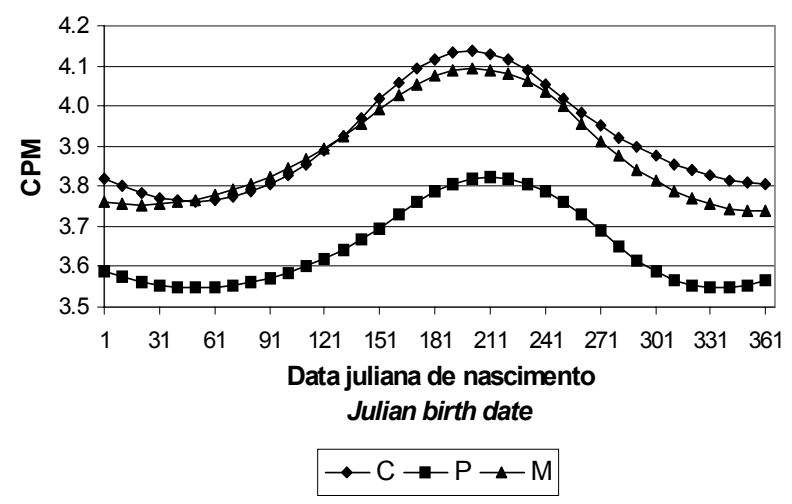

Figura 3 - Valores estimados para o ganho de peso do nascimento à desmama (GND) e para os escores de conformação $(C)$, precocidade $(P)$ e musculatura $(M)$ em função da data juliana de nascimento.

Figura 3 - Estimated values of weight gain from birth to weaning (GND) and for conformation (C), precocity (P), and muscling (M) scores as a function of Julian birth date. 


\section{Conclusões}

A análise dos dados permite concluir que a idade do bezerro, a idade da vaca e a data juliana do nascimento do bezerro são importantes fontes de variação nos escores de conformação, precocidade e musculatura à desmama, similarmente ao verificado para ganho de peso do nascimento à desmama. Com base na magnitude dos efeitos estimados, considerar essas fontes de variação ambiental nas avaliações genéticas para os escores de conformação, precocidade e musculatura aumentará a acurácia das estimativas dos valores genéticos dos animais.

Os escores de conformação, precocidade e musculatura à desmama apresentam efeitos heteróticos maternos de magnitude similar aos verificados para ganho de peso do nascimento à desmama. Entretanto, os efeitos heteróticos individuais para os escores são inferiores àqueles para o ganho do nascimento à desmama.

A magnitude da heterose materna e individual nos escores de conformação, precocidade e musculatura à desmama, indica que considerar a heterose nas avaliações genéticas para esses escores aumentará a acurácia das estimativas dos valores genéticos dos animais.

\section{Literatura citada}

BARCELOS, S.J.; LOBATO, J.F.P. Efeitos da época de nascimento no desenvolvimento de bezerros Hereford e suas cruzas. I. Peso ao nascer e ganho médio diário prédesmama. Revista Brasileira de Zootecnia, v.21, n.1, p.137-149, 1992.

BOCCHI, A.L.; FRIES, L.A.; ALBUQUERQUE, L.G. Efeito da idade da vaca e da data juliana do nascimento sobre o ganho médio diário de bezerros de corte durante o período prédesmame. In: REUNIAO ANUAL DA SOCIEDADE BRASILEIRA DE ZOOTECNIA, 36, 1999, Porto Alegre. Anais... Porto Alegre: SBZ/Gnosis, [1999] 154pag. CD-ROM. Melhoramento Animal. MEL-054.

CAMPOS, L.T.; SILVA, P.R.; FRIES, L.A. Fatores de correção para os efeitos ambientais que afetam o ganho de peso do nascimento à desmama em bovinos da raca Nelore. Coletânea de pesquisas inéditas sobre o zebu.Uberaba: Empresa de Pesquisa Agropecuária de Minas Gerais, 1989. p.108-123.

CARDOSO, F.F. Caracterização genética do desempenho do nascimento à desmama de bovinos Aberdeen Angus criados no Rio Grande do Sul. Pelotas: Universidade Federal de Pelotas, 1999. 116p. Dissertação (Mestrado em Zootecnia) - Universidade Federal de Pelotas, 1999.

CARDOSO, F.F.; CARDELLINO, R.A.; CAMPOS, L.T. Fatores ambientais sobre escores de avaliação visual à desmama em bezerros Angus criados no Rio Grande do Sul. Revista Brasileira de Zootecnia, v.30, n.2, p.318-325, 2001.

FRIES, L. A. Efeito da data juliana de nascimento sobre o peso a desmama em zebuínos. In: Simpósio Nacional de Melhoramento Genético, 1, 1996, Ribeirão Preto. Anais... Ribeirão
Preto: Sociedade Brasileira de Melhoramento Animal, 1996a. p. 249-251.

FRIES, L.A. Efeito da idade da vaca sobre o peso a desmama em zebuínos. In: Simpósio Nacional de Melhoramento Genético, 1., 1996, Ribeirão Preto. Anais... Ribeirão Preto: Sociedade Brasileira de Melhoramento Animal, 1996b. p.255-256.

FRIES, L.A.; JOHNSTON, D.J.; HEARNSHAW, H. et al. Evidence of epistatic effects on weaning weight in crossbred beef cattle. Asian-Australian Journal of Animal Science, v.13, B, Supplement, p. 242, 2000.

GREGORY, K.E.; CUNDIFF, L.V. Crossbreeding in beef cattle: evaluation of systems. Journal of Animal Science, v.51, n.5, p.1224-1242, 1980.

PAZ, C.C.P. Fatores ambientais e genéticos que afetam o ganho de peso pré-desmame em bovinos da raca Nelore. Jaboticabal: Universidade Estadual Paulista, 1997. 117p. Dissertação (Mestrado em Zootecnia) - Universidade Estadual Paulista, 1997.

PICCOLI, M.L. Estudo de curvas de crescimento de bovinos Polled Hereford criados em pastagem. Porto Alegre: Universidade Federal do Rio Grande do Sul, 1992. 212p. Dissertação (Mestrado em Zootecnia) - Universidade Federal do Rio Grande do Sul, 1992.

PITA, F.V.C.; ALBUQUERQUE, L.G.; FRIES, L.A. Utilização de escores visuais como indicadores da idade à maturidade sexual de novilhas Nelore. In: SIMPÓSIO NACIONAL DE MELHORAMENTO GENÉTICO, 2., 1998, Uberaba. Anais... Uberaba: Sociedade Brasileira de Melhoramento Animal, 1998. p. 383-384.

PONS, S.B; MILAGRES, J.C.; TEIXEIRA, N.M. Efeitos de fatores genéticos e de ambiente sobre o crescimento e o escore de conformação à desmama. Revista da Sociedade Brasileira de Zootecnia, v.18, n.5, p.391-401, 1989.

RESTLE, J.; VAZ, F.N.; FEIJÓ, G.L.D. et al. Características de carcaça de bovinos de corte inteiros ou castrados de diferentes composições raciais Charolês x Nelore. Revista Brasileira de Zootecnia, v.29, n.5, p.1371-1379, 2000.

ROSO, V.M.; FRIES, L.A. Componentes principais em bovinos da raça Polled Hereford à desmama e sobreano. Revista da Sociedade Brasileira de Zootecnia, v.24, n.5, p.728-735, 1995.

ROSO, V.M.; FRIES, L.A. Avaliação das heteroses materna e individual sobre o ganho de peso do nascimento ao desmame em bovinos Angus x Nelore. Revista Brasileira de Zootecnia, v.29, n.3, p.732-737, 2000.

SAS INSTITUTE. Statistical Analysis System. User's Guide. 3.ed. Cary: 1995. 530p.

TEIXEIRA, R.A. Heterose materna e individual sobre o ganho de peso pré-desmama em bovinos Nelore $x$ Hereford e Nelore $x$ Angus. Jaboticabal: Universidade Estadual Paulista, 2000. 75p. Dissertação (Mestrado em Zootecnia) - Universidade Estadual Paulista, 2000. 